\title{
Clinical effectiveness and micro-perfusion alteration of Jingui external lotion in patients with knee osteoarthritis: study protocol for a randomized controlled trial
}

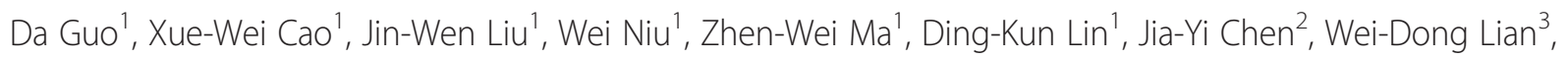
Wen-Wei Ouyang ${ }^{4}$ and Jun Liu ${ }^{1 *}$

\begin{abstract}
Background: Knee osteoarthritis is a major cause of disability in the aging population. Based on pathological, magnetic resonance imaging (MRI) and arthroscopy studies, progressive osteoarthritis involves all tissues of the joint and includes bone marrow lesions, synovial proliferation, fat pad inflammation, and high subchondral bone turnover. Recent research suggests that abnormal perfusion in bone marrow lesions, fat pads, and subchondral bone is associated with pain in knee osteoarthritis, and that dynamic contrast-enhanced MRI is a promising method for studying micro-perfusion alteration in knee osteoarthritis. Traditional Chinese Medicine approaches have been employed for thousands of years to relieve knee osteoarthritis pain. Among herbal medicines, the Jingui external lotion is the preferred and most commonly used method in China to reduce pain in patients with knee osteoarthritis; however, there is a lack of validated evidence for its effectiveness. The purpose of this study is to explore the effectiveness of Jingui external lotion for the management of painful knee osteoarthritis in a short-term study. In addition, we will assess micro-perfusion alteration in the patellar fat pad as well as the femur and tibia subchondral bone via dynamic contrast-enhanced MRI.
\end{abstract}

Methods/design: This trial is a randomized, controlled study. A total of 168 patients will be randomized into the following two groups: 1) the Jingui external lotion group (treatment group); and 2) the placebo lotion group (control group). In both groups, lotion fumigation and external washing of the patients' knees will be administered twice a day for 14 consecutive days. Follow-up will be at regular intervals during a 4-week period with a visual analog scale to assess pain, and additional characterization with the Western Ontario and McMaster Universities Index score; rescue medication will be recorded as the extent and time pattern. In addition, micro-perfusion alteration in the patellar fat pad, femur and tibia subchondral bone will be assessed via dynamic contrast-enhanced MRI.

Discussion: This study will provide clinical evidence of the efficacy of Jingui external lotion in treating knee osteoarthritis, and it will be the first randomized controlled trial to investigate micro-perfusion alteration of knee osteoarthritis with Traditional Chinese Medicine external lotion via dynamic contrast-enhanced MRI.

Trial registration: ClinicalTrials.gov identifier: ChiCTR-TRC-14004727; 31 May 2014.

Keywords: Knee osteoarthritis, Jingui external lotion, Clinical effectiveness, Micro-perfusion alteration, Dynamic contrast-enhanced MRI, Randomized controlled trial

\footnotetext{
* Correspondence: liujun.gdtcm@hotmail.com

'Department of Orthopedic Surgery, The Second School of Clinic Medicine, Guangzhou University of Chinese Medicine, No. 111 Dade Road, Guangzhou, Guangdong 510120, China

Full list of author information is available at the end of the article
} 


\section{Background}

Knee osteoarthritis (KOA) is a major cause of disability in the aging population and a burden on healthcare resources [1,2]. The risk of disability (defined as needing help walking or climbing stairs) attributable to $\mathrm{KOA}$ is as great as that attributable to cardiovascular disease and greater than that due to any other medical condition in elderly persons [3]. Pathologically, osteoarthritis (OA) is characterized by progressive loss of articular cartilage and new bone formation on conventional radiography. However, it is increasingly apparent, based on pathological, magnetic resonance imaging (MRI) and arthroscopy studies, that progressive OA involves all joint tissues including bone marrow lesions, synovial proliferation, fat pad inflammation, and high subchondral bone turnover [4-7]. This progressive joint failure causes pain, physical disability, and psychological distress, although many with structural and radiological changes consistent with $\mathrm{OA}$ are asymptomatic [8]. In recent years, more attention has been placed on micro-perfusion alteration in KOA. Studies [9-11] employed dynamic contrastenhanced (DCE) MRI to investigate the association between knee pain and periarticular perfusion variables. These results suggest that abnormal perfusion including bone marrow lesions, fat pad, and subchondral bone as detected by DCE MRI are associated with pain in KOA and that DCE MRI is a promising method for studying micro-perfusion alteration in KOA.

The aim of KOA treatment is to reduce pain, improve physical function, prevent disability, and enhance the quality of life over the short term. Therefore, as aging and comorbidities increase, a more convenient approach is urgently needed. In China, and increasingly worldwide, pharmacological therapy guidelines recommend topical medications as an alternative, adjunctive therapy, or even first-line therapy for KOA $[12,13]$. Before oral administration, topical treatments are usually recommended to relieve mild or moderate KOA pain because of their favorable safety profiles. To relieve KOA pain, herbal external therapy, including plasters, ointments, and lotions, have been used under the principle of syndrome differentiation based on Traditional Chinese Medicine (TCM) theory for thousands of years. External plasters have been reported to be very important therapies that induce muscle relaxation and invigorate blood circulation [14,15]. Jingui external lotion with fumigation and washing therapy has been employed as an effective prescription to relieve KOA pain or joint stiffness over decades, but no randomized controlled trial evidence has been published. Published Chinese literature has documented that Jingui external lotion is efficacious in treating KOA; however, these interventions have not been rigorously evaluated, and have been regarded to be less credible due to a lack of objective evidence. Therefore, we designed a randomized, single-blind, placebo-controlled trial to explore the effectiveness of Jingui external lotion for the management of painful KOA in a short-term study and the micro-perfusion alteration in the patellar fat pad, femur and tibia subchondral bone via DCE MRI.

\section{Methods/design}

\section{Study design}

This is a randomized, double-blind, placebo-controlled trial. The trial protocol has been approved by the Research Ethical Committee of Guangdong Provincial Hospital of TCM, (reference B2014-001-01). The trial will be conducted in accordance with the Helsinki Declaration and will be monitored by the trial agency at Guangdong Provincial Hospital of TCM.

\section{Recruitment and consent}

KOA patients scheduled for conservative treatment at the Outpatient Department of Orthopedic Surgery, Guang Dong Provincial Hospital of TCM, will be recruited, with a target sample size of 168 subjects. All candidates will go through a standardized interview process and receive more information about the study and the treatments. Written consents will be obtained. The purpose, procedures, and potential risks and benefits of the study will also be explained thoroughly to the participants. The more painful knee will be chosen as the study knee, and if both knees have the same symptoms we will choose the right side. The participants will be able to withdraw from the study at any time without consequence. The trial will be executed from March 2014 to March 2016 including enrollment and follow-up (Figure 1).

\section{Inclusion criteria}

Participants meeting the following criteria will be included:

- Meet the diagnostic criteria for KOA (American College of Rheumatology criteria [16])

- Aged from 50 to 80 years old

- Symptomatic KOA with pain of at least $20 \mathrm{~mm}$ on a 100-mm visual analogue scale (VAS)

- Grade 0 to 3 on The Kellgren-Lawrence grading system

- No serious medical history or severe vascular lesions on lower limbs

- No known drug allergies, including to the contrast agent, and no MRI contraindications

- Willingness to give written informed consent and willingness to participate in and comply with the study. 


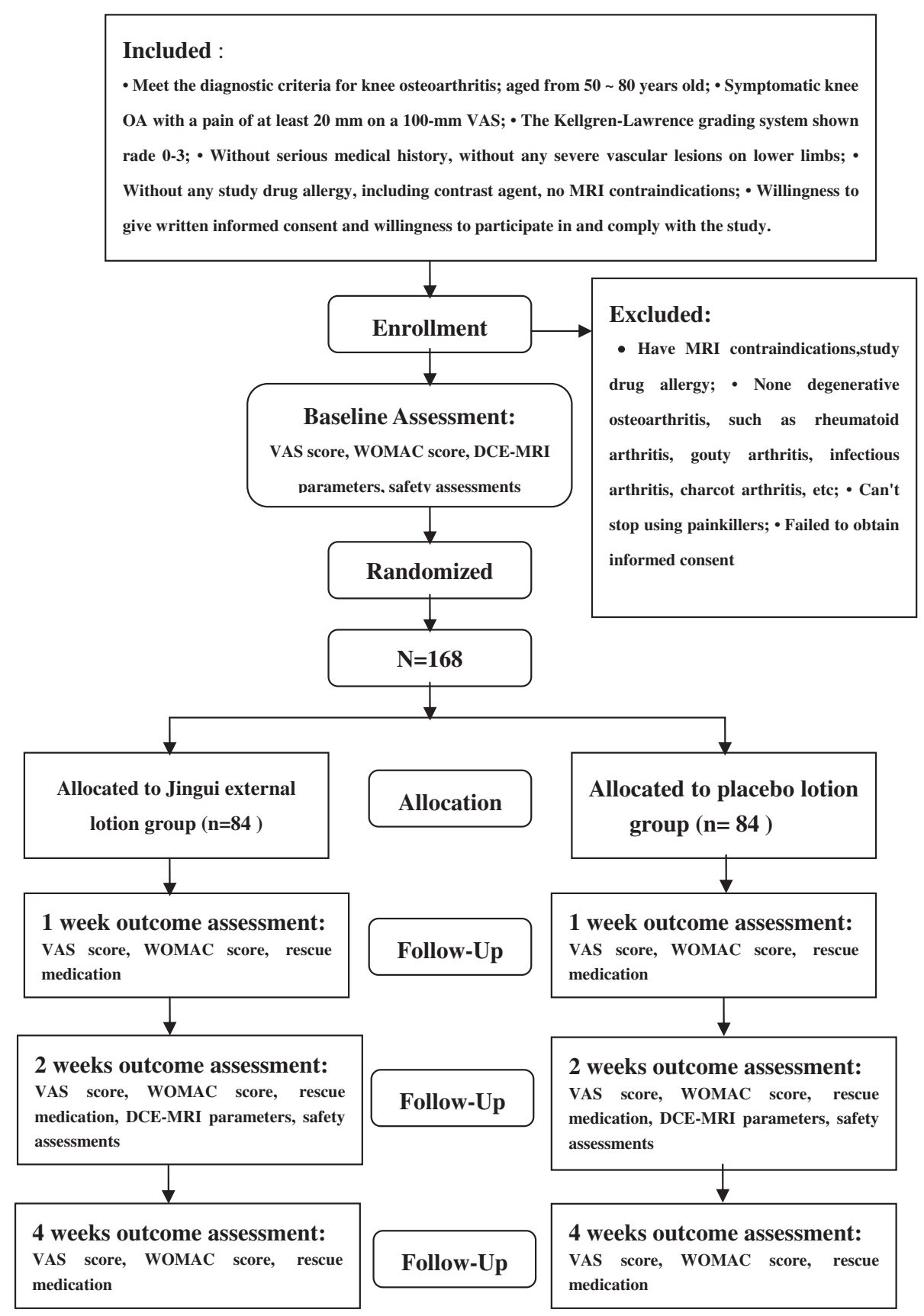

Figure 1 Study flow chart. DCE MRI, dynamic contrast-enhanced magnetic resonance imaging; OA, osteoarthritis; VAS, visual analogue scale; WOMAC, Western Ontario and McMaster Universities Index.

\section{Exclusion criteria}

Participants meeting one or more of the following criteria will be excluded:

- MRI contraindications, or study drug allergy

- Non-degenerative OA, such as rheumatoid arthritis, gouty arthritis, infectious arthritis, Charcot arthritis, and so forth
- Unable to discontinue use of painkillers

- Unwilling to give informed consent.

\section{Interventions}

Eligible patients will be randomized into the following two groups: the Jingui external lotion group (treatment group) and the placebo lotion group (control group). 
The enrolled patients will be administered lotions via fumigating and washing externally for 2 weeks on both knees. DCE MRI examination will be performed at baseline and 2 weeks after the intervention is completed. The participants will be given personal instructions for using the lotions by our research nurses, who will be trained before the study. Patient visits will be performed at baseline and at 1,2, and 4 weeks after treatment. Assessments including the VAS score for pain, Western Ontario and McMaster Universities Index (WOMAC) score and complications will be made during all four visits on the study knee.

The treatment group will receive Jingui external lotion, to be used twice a day (morning and evening, approximately every 12 hours and at the same time each day) for 14 consecutive days. The Jingui external lotion will be made according to the following procedure: a mixture of TCM (composed of Semiliquidambar cathayensis 60 g, Radix zanthoxyli 60 g, Radix aconiti kusenzoffii 30 g, Radix aconite 30 g, Tinospora sinensis merr 30 g, Erythrina indica lam $30 \mathrm{~g}$, Rheum officinale $30 \mathrm{~g}$, Cassia twig $30 \mathrm{~g}$ ) will be boiled in $300 \mathrm{ml}$ water, reduced to $150 \mathrm{ml}$ of liquid medicine, and cooled to approximately $40^{\circ} \mathrm{C}$. These hot liquid medicines will be administered as Jingui external lotion to the patients' knees.

The control group will receive placebo lotion containing a food coloring agent and a small amount of Rheum officinale that is identical to the treatment group in terms of color and odor. The manufacturing and treating procedure are the same as the treatment group.

The research nurses will be involved in the treatment procedure to ensure the correct treatment is administered and that the temperature of the lotions is tolerable to the patients. During the process, when patients experience moderate pain or VAS score over $30 \mathrm{~mm}$, ibuprofen sustained-release capsules will be administrated as rescue medication. Rescue medication consumption and time will be recorded in each randomized group when used. The patients will not be allowed to use other drugs aimed at treating KOA.

\section{Dynamic contrast-enhanced magnetic resonance imaging examination}

The subjects will receive DCE MRI on the study knee at baseline and 2 weeks after intervention using a $3.0 \mathrm{~T}$ scanner (Magnetom Tim Trio; Siemens Medical Solutions, Erlangen, Germany) with an eight-channel transmitreceive phased array knee coil (In vivo Corporation, Gainesville, FL, USA). The knee imaging protocol will consist of a sagittal three-dimensional high-resolution T1weighted fast low angle shot sequence with selective water excitation $(\mathrm{TR} / \mathrm{TE}=500 / 20 \mathrm{~ms}$; flip angle $=25 ; \mathrm{FOV}=$ $18.0 \mathrm{~cm}$; slice thickness $=3 \mathrm{~mm}$; matrix $=320 \times 240$; receiver bandwidth $=200 \mathrm{~Hz} /$ pixel) as well as a sagittal
T2-weighted fat-saturated spin echo $(\mathrm{TR} / \mathrm{TE}=4790 /$ $84 \mathrm{~ms} ; \mathrm{FOV}=18.0 \mathrm{~cm}$; slice thickness $=3 \mathrm{~mm}$; matrix = $320 \times 240$; receiver bandwidth $=130 \mathrm{~Hz} /$ pixel). The synovial membrane will be evaluated using DCE sagittal three-dimensional T1-weighted fast low angle shot sequence with the following parameters: TR/TE $=12 / 3.9 \mathrm{~ms}$, flip angle $=60 ; \mathrm{FOV}=15 \times 15 \mathrm{~cm}$, slice thickness $=5 \mathrm{~mm}$, matrix $=256 \times 128$, receiver bandwidth $=200 \mathrm{~Hz} /$ pixel, temporal resolution $=30$ seconds. This sequence will be acquired in contiguous 5-mm sagittal slices throughout the knee before, during and after intravenous bolus administration of double-dose contrast agent gadolinium diethylene triamine pentaacetic acid $(0.2 \mathrm{mg} / \mathrm{kg})$. Baseline precontrast static images as well as DCE images will be acquired after bolus injection $[17,18]$. The total acquisition time for the imaging protocol is 24 minutes.

Three-dimensional images of the knee joint will be rebuilt using a Leonardo workstation (Siemens Medical Solutions INC USA, 2501 North Barrington Rd, Hoffman Estates Il 60195). The sagittal DCE sequence images and the intensity-time curve will be attained with the mean curve function. Four similar subchondral rectangular regions of interest (pixel area of 30) will be selected in the medial and lateral compartment of the distal femur and tibia plateau as well as the infra-patellar fat pad and supra-patellar fat pad. Measurement of these regions will be performed in terms of the enhanced rate and maximum upslope.

\section{Outcome measures}

\section{Primary outcome measure}

The primary efficacy endpoint of the study will be VAS, which is a pain score ranging from $0 \mathrm{~mm}$ (no pain) to $100 \mathrm{~mm}$ (worst pain ever experienced) [19], measured during all the assessment visits (baseline and 1-, 2-, and 4-week follow-up). The VAS score is usually a horizontal line, $100 \mathrm{~mm}$ in length, anchored by word descriptors at each end. Patients mark the point of their current pain on the line. The VAS score is then determined by measuring in millimeters from the left end of the line to the point that the patient marked.

\section{Secondary outcome measure}

The secondary efficacy endpoint of the study will be rescue medication, WOMAC score [20] and microperfusion alteration in the patellar fat pad, femur and tibia subchondral bone.

Rescue medication complicates the interpretation of trial results by having an effect on the trial outcome. It is also possible to use rescue as a trial outcome. This is common in trials of treatments for asthma, where use of $\beta_{2}$-agonists for rescue therapy may be a secondary trial outcome [21]. Rescue medication in this trial is Ibuprofen, which is a short-term nonsteroidal anti-inflammatory 
drug. We assume that the use of rescue medication varies with time in subjects in response to worsening of their disease. Consumption and time the subjects use rescue medication will be recorded in each randomized group.

The WOMAC score is a widely used proprietary set of standardized questionnaires used by health professionals to evaluate the condition of patients with osteoarthritis of the knee and hip, including pain, stiffness, and physical functioning of the joints. The index measures five items for pain (score range 0 to 20), two for stiffness (score range 0 to 8 ), and 17 for physical function (score range 0 to 68). It will be measured during all the assessment visits (baseline and 1-, 2-, and 4-week follow-up).

To assess micro-perfusion alteration, two parameters will be used: 1) maximum upslope represents the initial uptake of gadolinium into the region measured by finding the fastest uptake in the first few minutes after injection; 2) an enhanced rate represents the efflux rate constant from the extravascular extracellular space to plasma, which provides an index of venous hypertension and represents the ratio of the permeability surface area product over the extravascular extracellular space. With the mean curve function, maximum upslope and enhanced rate in the distal femur and tibia plateau subchondral regions of interest, and the infra-patellar fat pad and supra-patellar fat pad regions of interest will be measured and assessed.

\section{Safety assessments}

All subjects will be questioned about adverse events during the treatment at each visit, and all adverse events reported will be analyzed, regardless of the investigators' assessments of causality. Safety will be assessed by complete blood cell count, erythrocyte sedimentation rate, blood chemistry, and urinalysis.

\section{Randomization and blinding}

Random assignment will be performed after consent is obtained using a computer-generated, blocked randomallocation sequence with a 1:1 ratio. The trial will be blinded to both patients and assessor. We will designate an independent researcher who is blinded to the interventions to be the assessor.

\section{Sample size}

Calculation of sample size is based on previous studies assessing the efficacy and safety of Weishi Bitong Xifang fumigation for mild and moderate $\mathrm{KOA}$ in patients [22]. Group sample sizes of 73 and 73 achieve $90 \%$ power to detect a difference of 0.7 between the null hypothesis that both group means are $30 \mathrm{~mm}$ (VAS score uses a 100-mm linear measure) and the alternative hypothesis that the mean for the placebo group is 23 with estimated group standard deviations of 12 and 13 and with a significance level (alpha) of 0.05 using a two-sided test. The number of patients actually provides less than $90 \%$ power, assuming a withdrawal rate of $20 \%$. Therefore, we will recruit a total of 168 patients; 84 patients in each group.

\section{Statistical analysis}

The data will be collected and analyzed according to the intention-to-treat principle. We will compare baseline characteristics in both groups. Efficacy analyses will be performed for both the intent-to-treat population and per-protocol population. The intent-to-treat population will consist of all randomized subjects who have been administered at least one treatment. In a per-protocol analysis, only patients who complete the entire clinical trial according to the protocol are counted in the final results. Primary outcome will be compared between both groups. The extent and time pattern of rescue medication will be reported in each randomized group; when comparing the underlying outcomes of the rescue medication, we assume the use of rescue medication varies with time, to allow for dependence within patients, so we adopt a multilevel regression approach with rescue as a time-dependent covariate [23]. Microperfusion alteration outcome will be compared using Student's $t$-test. All statistical analyses will be performed using SAS 9.2 software (SAS Institute Inc, Cary, NC, USA). All statistical tests will be two-sided, and the level of significance will be set at 0.05 .

\section{Discussion}

This study will provide clinical evidence on the efficacy of Jingui external lotion in treating KOA, and it will be the first randomized controlled trial to investigate micro-perfusion alteration of KOA with TCM external lotion using a DCE MRI technique. In TCM, KOA development is attributed to deficiency of the liver and kidney, and deficiency of qi and blood, attack of windcold-dampness evils, obstruction of channels and collaterals, qi and blood stagnation [24]. Thus, TCM that can promote blood circulation, remove blood stasis, and activate meridians to stop pain may be effective for $\mathrm{KOA}$ [13]. Fumigation and washing of TCM has recently been considered an inexpensive and convenient therapeutic approach for KOA [25]. Jingui external lotion is a classical and well-known external therapy in the orthopedic departments of TCM and has been demonstrated to provide a satisfactory effect for KOA in the clinic. Welldesigned randomized controlled trials are needed to examine the efficacy of TCM treatments for OA.

It has been revealed that Chinese medicine promoting circulation and removing stasis could reduce intraosseous pressure [26]. Intraosseous hypertension has been reported to have an association with deep pain in 
patients with KOA [27]. To date, there is a lack of evidence to support the hypothesis that Chinese medicine promotes circulation; therefore, we will employ a DCE MRI technique to study micro-perfusion alteration, with the aim of attaining objective evidence of Jingui external lotion effectiveness in the treatment of patients with KOA.

\section{Trial status}

Recruitment commenced in March 2014, and the trial is scheduled to end in March 2016.

\section{Abbreviations}

DCE: dynamic contrast-enhanced; KOA: knee osteoarthritis; MRI: magnetic resonance imaging; OA: osteoarthritis; TCM: Traditional Chinese Medicine; VAS: visual analogue scale; WOMAC: Western Ontario and McMaster Universities Index.

\section{Competing interests}

The authors declare that they have no competing interests.

\section{Authors' contributions}

$J \mathrm{~L}$ conceived the study and prepared the initial protocol, and was in charge of coordination. DG drafted the manuscript and participated in the study design. JL, DG, XWC, JWL WN, ZWM, DKL, WDL and JYC contributed to the creation of the Manual of Procedures, implementation of the study protocol and acquisition of data. WWO contributed to the statistical analysis section. All authors read and approved the final manuscript.

\section{Acknowledgements \\ This study was funded by the Specialized Research Fund for Chinese medicine of Guangdong Provincial Hospital of TCM (No. E42701, No. YK2013B2N20), Science and Technology Planning Project of Guangdong Province, China (No. 2014.807), the Administration of Traditional Chinese Medicine of Guangdong Province in China (No. 20123006), the National Natural Science Foundation of China (No. 81473698), and Project of Guangdong Provincial Department of Finance (No. 2013KT1493, No. 2012KT1013).}

\section{Author details}

'Department of Orthopedic Surgery, The Second School of Clinic Medicine, Guangzhou University of Chinese Medicine, No. 111 Dade Road, Guangzhou, Guangdong 510120, China. ²Department of Orthopedic Surgery, Hospital of Traditional Chinese Medicine of Zhongshan, No. 3 Xunkang Road, Zhongshan, Guangdong 528400, China. ${ }^{3}$ Department of Orthopedic Surgery, Hospital of Traditional Chinese Medicine of Meizhou, No. 35 Meishong Road, Meizhou, Guangdong 514000, China. ${ }^{4}$ Department of Statistical Secretary, The Second School of Clinic Medicine, Guangzhou University of Chinese Medicine, No. 111 Dade Road, Guangzhou, Guangdong 510120, China.

\section{Received: 16 September 2014 Accepted: 17 March 2015}

\section{Published online: 28 March 2015}

\section{References}

1. Bijlsma JW, Berenbaum F, Lafeber FP. Osteoarthritis: an update with relevance for clinical practice. Lancet. 2011;377:2115-26.

2. Peat G, McCarney R, Croft P. Knee pain and osteoarthritis in older adults: a review of community burden and current use of primary health care. Ann Rheum Dis. 2001;60:91-7.

3. Guccione AA, Felson DT, Anderson JJ, Anthony JM, Zhang Y, Wilson PW, et al. The effects of specific medical conditions on the functional limitations of elders in the Framingham Study. Am J Public Health. 1994;84:351-8.

4. Abramson SB, Attur M. Developments in the scientific understanding of osteoarthritis. Arthritis Res Ther. 2009;11:227.

5. Conaghan PG, Felson D, Gold G, Lohmander S, Totterman S, Altman R. MRI and non-cartilaginous structures in knee osteoarthritis. Osteoarthritis Cartilage. 2006;14(Suppl A):A87-94.
6. Ayral X, Pickering EH, Woodworth TG, Mackillop N, Dougados M. Synovitis: a potential predictive factor of structural progression of medial tibiofemoral knee osteoarthritis - results of a 1 year longitudinal arthroscopic study in 422 patients. Osteoarthritis Cartilage. 2005;13:361-7.

7. D'Agostino MA, Conaghan P, Le Bars M, Baron G, Grassi W, Martin-Mola E, et al. EULAR report on the use of ultrasonography in painful knee osteoarthritis. Part 1: prevalence of inflammation in osteoarthritis. Ann Rheum Dis. 2005;64:1703-9.

8. Hannan MT, Felson DT, Pincus T. Analysis of the discordance between radiographic changes and knee pain in osteoarthritis of the knee. J Rheumatol. 2000;27:1513-7.

9. Seah S, Wheaton D, Li L, Dyke JP, Talmo C, Harvey WF, et al. The relationship of tibial bone perfusion to pain in knee osteoarthritis. Osteoarthritis Cartilage. 2012;20:1527-33.

10. Aaron RK, Dyke JP, Ciombor DM, Ballon D, Lee J, Jung E, et al. Perfusion abnormalities in subchondral bone associated with marrow edema, osteoarthritis, and avascular necrosis. Ann N Y Acad Sci. 2007;1117:124-37.

11. Krasnokutsky S, Belitskaya-Lévy I, Bencardino J, Samuels J, Attur M, Regatte $R$, et al. Quantitative magnetic resonance imaging evidence of synovial proliferation is associated with radiographic severity of knee osteoarthritis. Arthritis Rheum. 2011;63:2983-91.

12. Zhang W, Nuki G, Moskowitz RW, Abramson S, Altman RD, Arden NK, et al. OARSI recommendations for the management of hip and knee osteoarthritis. Part III: changes in evidence following systematic cumulative update of research published through January 2009. Osteoarthritis Cartilage. 2010;18:476-99.

13. China State Administration of Traditional Chinese Medicine. Clinical path of traditional Chinese medicine 22 professional 95 diseases. Beijing, China: China Traditional Chinese Medicine Press; 2011.

14. Wang X, Cao Y, Pang J, Du J, Guo C, Liu T, et al. Traditional Chinese herbal patch for short-term management of knee osteoarthritis: a randomized, double-blind, placebo-controlled trial. Evidence Based Comp Altern Med. 2012;2012:171706.

15. Li C, Frangione V, Rovati S, Zheng Q. Diclofenacepolamine medicated plaster in the treatment of minor soft tissue injuries: a multicenter randomized controlled trial. Curr Med Res Opin. 2013;29:1137-46.

16. Belo JN, Berger MY, Koes BW, Bierma-Zeinstra SM. The prognostic value of the clinical ACR classification criteria of knee osteoarthritis for persisting knee complaints and increase of disability in general practice. Osteoarthritis Cartilage. 2009;17:1288-92.

17. Fernandez-Madrid F, Karvonen RL, Teitge RA, Miller PR, An T, Negendank WG. Synovial thickening detected by MR imaging in osteoarthritis of the knee confirmed by biopsy as synovitis. Magn Reson Imaging. 1995;13:177-83.

18. Loeuille D, Chary-Valckenaere I, Champigneulle J, Rat AC, Toussaint F, Pinzano-Watrin A, et al. Macroscopic and microscopic features of synovial membrane inflammation in the osteoarthritic knee: correlating magnetic resonance imaging findings with disease severity. Arthritis Rheum. 2005;52:3492-501.

19. Trijau S, Avouac J, Escalas C, Gossec L, Dougados M. Influence of flare design on symptomatic efficacy of non-steroidal anti-inflammatory drugs in osteoarthritis: a meta-analysis of randomized placebo-controlled trials. Osteoarthritis Cartilage. 2010;18:1012-8.

20. Faik A, Benbouazza K, Amine B, Maaroufi H, Bahiri R, Lazrak N, et al. Translation and validation of Moroccan Western Ontario and McMaster Universities (WOMAC) osteoarthritis index in knee osteoarthritis. Rheumatol Int. 2008;28:677-83.

21. Pauwels RA, Löfdahl CG, Postma DS, Tattersfield AE, O'Byrne P, Barnes PJ, et al. Effect of inhaled formoterol and budesonide on exacerbations of asthma. Formoterol and Corticosteroids Establishing Therapy (FACET) International Study Group. N Engl J Med. 1997;337:1405-11.

22. Zhang H, Liu T, Li F. A random, case-control study on the efficacy and safety of Weishi Bitong Xifang fumigation for mild and moderate knee osteoarthritis patients. Int J Rheum Dis. 2013. doi:10.1111/1756-185X.12165

23. White IR, Bamias C, Hardy P, Pocock S, Warner J. Randomized clinical trials with added rescue medication: some approaches to their analysis and interpretation. Stat Med. 2001;20:2995-3008.

24. Wang HM, Liu JN, Zhao Y. Progress on integrated Chinese and Western medicine in the treatment of osteoarthritis. Chin J Integr Med. 2010;16:378-84.

25. Zhou SW, Shen Q, Liao YX. Clinical study of treating knee osteoarthritis (Bi syndrome of knee) by massage combined Chinese materia medica footbath 
fumigation and washing. Zhongguo Zhong Xi Yi Jie He Za Zhi. 2012;32:1060-3.

26. Jiwei W, Shicong F, Weibin S. The influence of traditional Chinese drugs on para genual intra osseous pressure in experimental osteoarthritis. J Tradit Chin Orthop Traumatol. 1997;:43-4.

27. Simkin PA. Bone pain and pressure in osteoarthritic joints. Novartis Found Symp. 2004;260:179-86.

Submit your next manuscript to BioMed Central and take full advantage of:

- Convenient online submission

- Thorough peer review

- No space constraints or color figure charges

- Immediate publication on acceptance

- Inclusion in PubMed, CAS, Scopus and Google Scholar

- Research which is freely available for redistribution 